\title{
Application of Numerical Methods for the Analysis of Incremental Oil
}

\author{
Oloyede Olaoye, Oluwaseun Taiwo*, Olalekan Olafuyi
}

Nigeria

*Corresponding Author: Oluwaseun Taiwo, Nigeria

\begin{abstract}
Incremental oil values of Enhanced Oil Recovery projects have been obtained in the past using the Decline Curve Analysis and the Trapezoidal rule, with the former posing a challenge of inaccuracy in shifting while the latter approximates the area under a curve with a straight line segment. This has substantially undermine the accuracy and significance of the incremental oil values obtained by these methods.

In this work, Cubic spline was used to numerically model rate-time curves and Thomas algorithm was used to solve the resulting tri-diagonal system of equations. Also, Simpson quadrature was applied to the respective rate-time data and the concept of finite difference was introduced to account for the error term. Experimental data was used for the analysis.

Result showed a more accurate predictions of Incremental oil by the Cubic spline and Simpson quadrature as compared to Trapezoidal rule and Decline curve. Since, incremental oil values rightly measures the success of any EOR project by estimating accurate volumes of oil produced via such displacement mechanism, it imply that adopting the Natural cubic spline and Simpson quadrature will aid better estimations of the economic values of projects and effective management decision making.
\end{abstract}

Keywords: EOR, Cubic spline, Simpson quadrature, Trapezoidal rule and Decline curve

\section{INTRODUCTION}

A universal technical measure of the success of an Enhanced oil recovery (EOR) project is the amount of the incremental oil recovered. Figure.1below defines incremental oil. Imagine a field, reservoir, or a well whose oil rate is declining from $\mathbf{A}$ to $\mathbf{B}$. At $\mathbf{B}$, an EOR project was initiated, and if successful, the rate should show a deviation from the projected decline after $\mathbf{B}$. Incremental oil been the difference between what was actually recovered, $\mathbf{B}$ to $\mathbf{D}$ and what would have been recovered had the process not been initiated, $\mathbf{B}$ to $\mathbf{C}$.is estimated by the area under rate-time curve; the incremental oil recovered is therefore the shaded region.

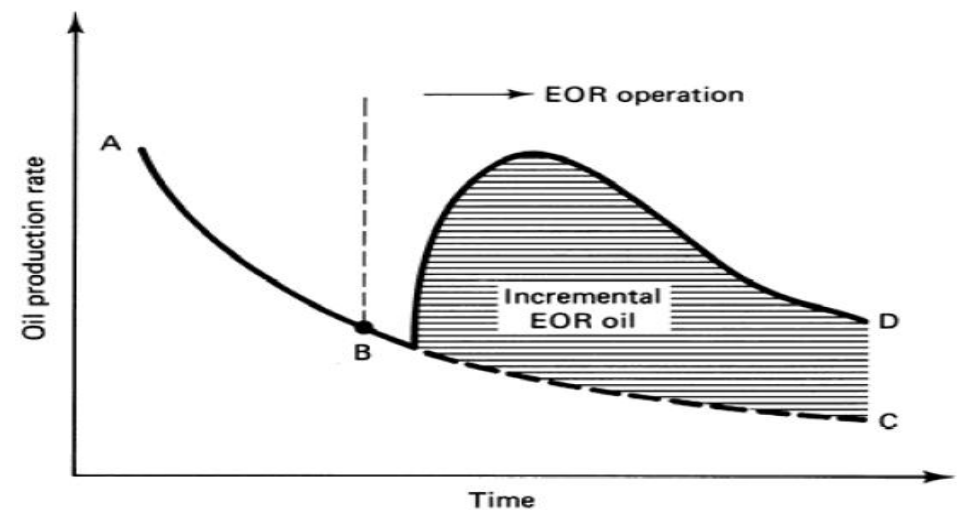

Figure1. Incremental Oil Recovery from Typical EOR Response curve ${ }^{[1]}$

Consequently, the additional amount of hydrocarbon in place produced through the successful application of an EOR technique, in the excess of the existing (conventional) process - primary or secondary recovery processes of operation - is referred to, technically as the Incremental oil. 
Estimating the incremental oil from an EOR process involves integration to evaluate the area under the rate-time curve. Ways of achieving this over the years in the industry ranges from the use of models to graphical procedures. One of the model is the Decline curve method and one of the graphical procedures is the Trapezoidal rule. The Decline curve is an effective tool used in predicting well performance and reservoir life based on real life data. But, its adoption in estimating incremental oil have shown little success in the past, as its results have shown to be highly erroneous. This is attributed to the challenge of inaccuracy in shifting ${ }^{[2]}$ while, the Trapezoidal rule approximates of the integral under a curve with that under a straight line segment ${ }^{[3]}$. These challenges have substantially impair the accuracy of incremental oil predictions obtained from EOR projects.

To minimize these errors, this paper propose to adapt other numerical methods, notably the natural cubic spline and Simpson quadrature in modeling EOR curves andpredictingthe incremental oil recovered from such displacement mechanism. Furthermore, it seeks to comparatively analyze the performance these numerical techniques with pre-existing methodologies in order to determine their level of accuracy and emerge with a more robust approach to incremental Oil prediction.

Some experimental works where data can be obtained for use includes the works of Onuoha and Olafuyi, 2013, Orivri et al, 2014, Atsenuwa et al, 2014, Yebu et al, Uwari et al, Kharma et al, Taiwo and Olafuyi in $2015^{[4-9]}$. And some other works of Taiwo et al in 2016, Mamudu et al 2016 , Onuoha et al 2017, Ojukwu et al2018, Madugu et al 2018, and Taiwo et al $2019^{[10-15]}$ can were also of great contributions to this work in estimating the incremental oil from EOR.

\section{METHODS}

\subsection{Trapezoidal Rule}

The trapezoidal is the first of Newton-Cotes integration formulae. Trapezoidal Rule is an already established numerical integration formula which involves the use of the integral under a straight line to approximate the integral under a curve. The respective mathematical model for the rule is clearly outlined below.

$I=\int_{a}^{b} f x d x$

For a single strip (two ordinates)

$I=\frac{h}{2}\left\lfloor f\left(x_{0}\right)+f\left(x_{1}\right)\right\rfloor-\frac{h^{3}}{12} f^{\prime \prime}(x)$

For multiple strips ( $n>2$ ordinates)

$I=\frac{h}{2}\left[f\left(x_{0}\right)+2\left(f\left(x_{1}\right)+f\left(x_{2}\right)+f\left(x_{3}\right)+\cdots+f\left(x_{n-1}\right)\right)+f\left(x_{n}\right)\right]-\frac{h^{3}}{12} f^{\prime \prime}(x)$

Error $=-\frac{h^{3}}{12} n f^{\prime \prime}(x)$

Where $I=$ Area under the graph

\subsection{The Simpson's Rule}

One drawback of the trapezoidal rule is that its error is related to the second derivation. Therefore more complicated approximations formulas can improve the accuracy for curves, these includes using $2^{\text {nd }}$ and $3^{\text {rd }}$ order polynomial. There are two versions of this rule as exists.

\subsection{The Simpson's $1 / 3^{\text {th }}$ Rule}

In this rule, the function is approximated by a second-order degree polynomial between successive points. It corresponds to using second-order Lagrange polynomials in modelling data points.

Mathematically,

$\boldsymbol{I}=\frac{h}{3}\left[f\left(x_{0}\right)+4\left[f\left(x_{1}\right)+f\left(x_{3}\right) \ldots+f\left(x_{2 n-1}\right)\right]+2\left\lfloor f\left(x_{2}\right)+f\left(x_{4}\right) \ldots+f\left(x_{2 n-2}\right)\right\rfloor+f\left(x_{n}\right)\right]$

The error associated with this rule is given by the model;

Error $=-\frac{n h^{5}}{90} f^{4}(x)$ 


\subsection{The Simpson's Three-Eight Rules}

This rule corresponds to using third-order polynomial to fit four points. Integrating over the four points gives; the model is given as shown below.

$\left.\boldsymbol{I}=\frac{3 h}{8} \mid f\left(x_{0}\right)+3\left[f\left(x_{1}\right)+\cdots+f\left(x_{3 n-1}\right)\right]+2\left(f\left(x_{3}\right) \ldots+f\left(x_{3 n-3}\right)\right)+f\left(x_{3 n}\right)\right]-\frac{n h^{5}}{80} f^{4}(x)$

The error associated with this rule is given by the model;

Error $=-\frac{n h}{80} f^{4}(x)$

\subsection{Numerical Error}

Past works on these quadratures, have shown that they have tendencies to under or over predict sometimes, depending on the data architecture. Therefore, in view of this, it becomes pertinent that the differentials for the respective quadrature be accounted for in order to mitigate any of these cases occurring and consequently ensuring a more accurate incremental oil result from displacement mechanisms.

Since laboratory rate -time values mimicking that obtainable from the field were considered, this paper utilizes the numerical differentiation technique, known as the Finite difference to account for the error as shown below

$\frac{d y}{d x}=\frac{\Delta f_{i}}{\Delta x}$

This can be extended to the second and fourth differentials in order to fully account for the error in the Trapezoidal and Simpson's rules respectively.

$\frac{d^{2} y}{d x^{2}}=\frac{f_{i+2}-2 f_{i+1}+f_{i}}{\Delta x^{2}}$

$\frac{d^{4} y}{d x^{4}}=\frac{\Delta^{4} f_{i}}{\Delta x^{4}}=\frac{f_{i+5}-4 f_{i+3}+6 f_{i+2}-4 f_{i+1}+f_{i}}{\Delta x^{4}}$

Therefore the Newton forward difference table would be drawn for each data.

\subsection{Cubic Spline Interpolation}

Real world numerical data is usually difficult to analyze. Any function which would effectively correlate the data would be difficult to obtain and highly unwieldy. To this end, the idea of the cubic spline was developed. Using this process, a series of unique cubic polynomials are fitted between each of the data points, with the stipulation that the curve obtained be continuous and appear smooth. The mathematical spline is similar in principle. The points, in this case, are numerical data. The weights are the coefficients on the cubic polynomials used to interpolate the data. These coefficients 'bend' the line so that it passes through each of the data points without any erratic behavior or breaks in continuity ${ }^{[16]}$

\subsection{Cubic Spline Model Formulation}

Figure 2 below depicts a cubic spline that spans $n$ knots. Denoting $f_{i, i+1}(x)$ as the cubic polynomial that spans the interval between knots $i$ and $i+1$, we note that the spline is a piecewise cubic curve, assembled together to form the $n-1$ cubics, $f_{1,2}(x), f_{2,3}(x), \ldots \ldots, f_{n-1, n}(x)$, all of which have different coefficients.



Figure2. Notation used to derive quadratic splines. 
Notice that there are $n$ interval and $n+1$ data points.

Using Lagrange's two-point interpolation, the second derivative which is a linear function can be expressed as:

$f^{\prime \prime}(x)=\left(\frac{x_{i+1}-x}{x_{i+1}-x_{i}}\right) f^{\prime \prime}\left(x_{i}\right)+\left(\frac{x-x_{i}}{x_{i+1}-x_{i}}\right) f^{\prime \prime}\left(x_{i+1}\right) \quad$ for $x_{i} \leq x \leq x_{i+1}$

Denoting the second derivative of the knot at $i$ by $k_{i}$ and with the condition of its continuity(from one spline to the successive), it's required that;

$f_{i-1,1}^{\prime \prime}\left(x_{i}\right)=f_{i+1,1}^{\prime \prime}\left(x_{i}\right)=k_{i}$

Integrating twice with respect to $x$ and with the condition that $f(x)$ is required to pass through $\left(x_{i}, y_{i}\right)$ and $\left(x_{i+1}, y_{i+1}\right)$ gives:

$f_{i, i+1}(x)$

$=\frac{k_{i}}{6}\left[\frac{\left(x-x_{i+1}\right)^{3}}{x_{i}-x_{i+1}}-\left(x-x_{i+1}\right)\left(x_{i}-x_{i+i}\right)\right]-\frac{k_{i+1}}{6}\left[\frac{\left(x-x_{i}\right)^{3}}{x_{i}-x_{i+1}}-\left(x-x_{i}\right)\left(x_{i}-x_{i+i}\right)\right]$

$+\frac{y_{i}\left(x-x_{i+1}\right)-y_{i+1}\left(x-x_{i}\right)}{x_{i}-x_{i+1}}$

The above model derives the cubic relationship between two successive data in a given data set. Therefore, this can be adapted to rate-time data to give the interpolant between two rates in successive time interval by the model;

$f_{i, i+1}(t)=\frac{k_{i}}{6}\left[\frac{\left(t-t_{i+1}\right)^{3}}{t_{i}-t_{i+1}}-\left(t-t_{i+1}\right)\left(t_{i}-t_{i+1}\right)\right]-\frac{k_{i+1}}{6}\left[\frac{\left(t-t_{i}\right)^{3}}{t_{i}-t_{i+1}}-\left(t-t_{i}\right)\left(t_{i}-t_{i+1}\right)\right]$

$+\frac{q_{i}\left(t-t_{i+1}\right)-q_{i+1}\left(t-t_{i}\right)}{t_{i}-t_{i+1}}$

Consequent upon this, there must be some formulations to determine the second derivatives $\left(k_{i}\right)$ at the interior knots. This leads to yet unused condition of continuity of the first derivative with which the equations of curvatures are formulated.

\subsection{Equations of Curvatures}

Here, we note that the second derivatives $\left(k_{i}\right)$ of the spline at the interior knots are found from the slop continuity conditions:

$f_{i, i-1}^{\prime}\left(x_{i}\right)=f_{i, i+1}^{\prime}\left(x_{i}\right) \quad i=1,2,3, \ldots \ldots, n-1$

Applying the conditions given by continuity between successive splinesresults in the following simultaneous equations:

$k_{i-1}\left(x_{i-1}-x_{i}\right)+2 k_{i}\left(x_{i-1}-x_{i+1}\right)+k_{i+1}\left(x_{i}-x_{i+1}\right)=6\left[\frac{y_{i-1}-y_{i}}{x_{i-1}-x_{i}}-\frac{y_{i}-y_{i+1}}{x_{i}-x_{i+1}}\right] i=2,3, \ldots n-1$

But if the data are evenly spaced at interval $(h)$, then we can write:

$h=x_{i-1}-x_{i}=x_{i}-x_{i+1}$

Then, Eq. (3.9) becomes:

$k_{i-1}+4 k_{i}+k_{i+1}=\frac{6}{h^{2}}\left[y_{i-1}-2 y_{i}+y_{i+1}\right] i=2,3, \ldots, n-1$

Eq. (19) is a set of $n-2$ linear simultaneous equations for the $n$ derivatives $\left(k_{i}\right)$, and when these are known, the spline function approximation formed bythe set of functions in Eq.(15) defined over the consecutive intervals $x_{i} \leq x \leq x_{i+1}$ can be constructed. It is crucial to the practical use of splines that this system of equations being nonsingular,and that an extremely efficient algorithm be available to solve it.In this reseach,Thomas Algorithmwas the solution method for the Triadiagonal system of equation resulting from Eqn19above. ${ }^{[17]}$

As the of values of $k_{1}$ and $k_{n}$ cannot be found from the condition that $f^{\prime}(x)$ is continuous across the nodes $x_{1}$ and $x_{n}$, these values must be specified as additional conditions. The choice of values for $k_{1}$ and $k_{n}$ prescribed as end conditions must be made intuitively, based on the way the data points 
indicate the interpulated curve is most likely to behave (be extrapolated) beyond the end points of the interval $x_{1} \leq x \leq x_{n}$. We used linear boundary condition in this research $\left(k_{1}=k_{n}=0 \text {. }\right)^{[18]}$

\subsection{End or Boundary Conditions}

Natural or linear spline end conditions.

This choice of end condition involves setting

$k_{1}=k_{n}=0$.

\section{APPLICATION}

Therefore ,Incremental oil is defined mathematically as given below;

Incremental oil $=\int_{a}^{b}($ Composite function $) d t-\int_{a}^{b} f(t) d t$

Where the Composite function= Cubic spline/Simpson rule/Trapezoidal rule.

$\int_{a}^{b} f(t) d t=$ Equation of projected decline

The equation for projected decline was determine by obtaining trendline for each cases study using Excel software.The resulting estimation from the composite functions were subtracted from that of the trendline in order to ascertain the incremental oil.Furthermore,in predicting the Incremental oil using the Decline curve,the economic limit for each experimental process was assumed to be the last rate prior to the EOR process execution.

The rate - time curves considered are shown below:

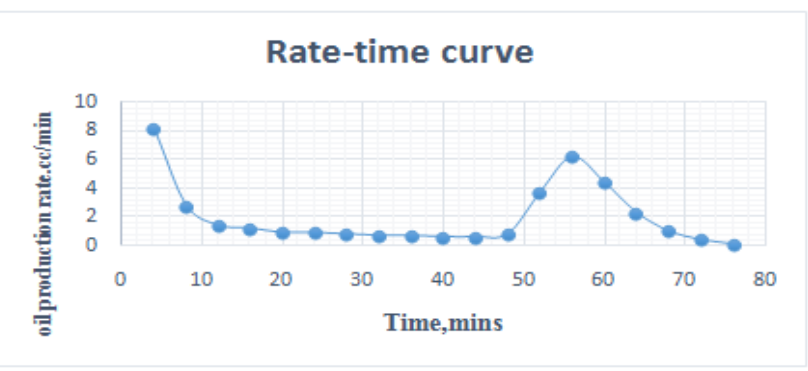

Figure3. Shows the rate-time profile for the polymer flooding process

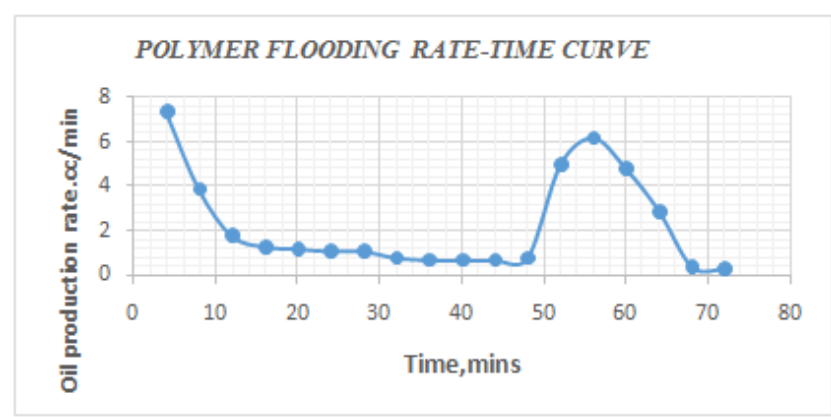

Figure4. Shows the rate-time profile for the polymer flooding process

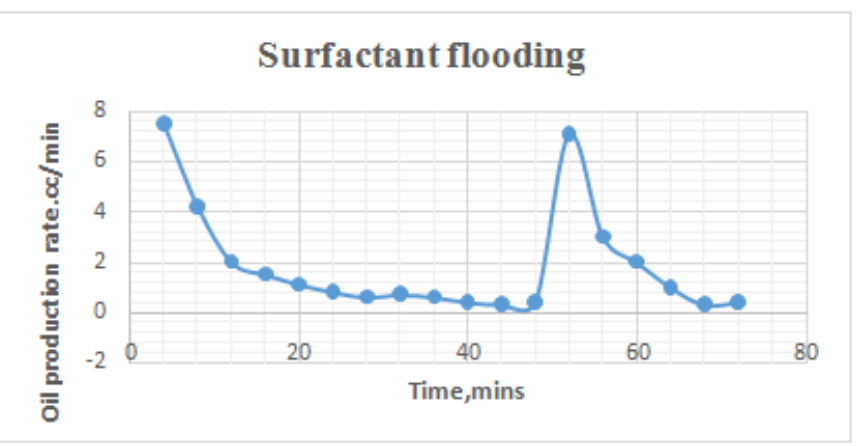

Figure5. Shows the rate-time profile for the surfactant flooding process 


\section{RESULT AND ANALYSIS}

This paper seeks to obtain accurate incremental oil prediction from successful EOR processes. Shown below is the outcome of our applied models (Natural cubic spline and Simpson rule) and their comparative performance with exiting methodologies (Decline curve and Trapezoidal)

\section{Case One: Polymer Flooding}



Figure6. Incremental predictions for the polymer flooding

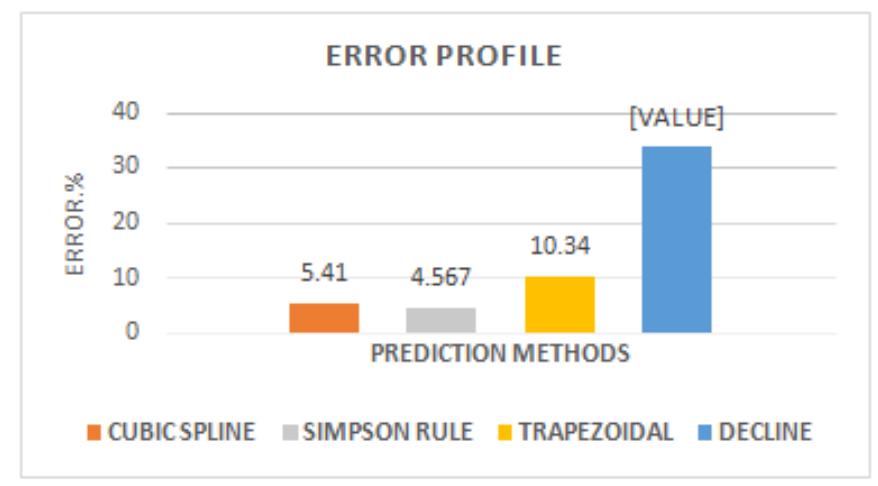

Figure6b. Error profile for polymer flooding case study

\section{Case Two: Polymer Flooding}



Figure7. Incremental oil prediction for the polymer flooding case study



Figure7b. Error profile for polymer flooding case study 


\section{Case Three: Alkaline Surfactant Polymer Flooding}

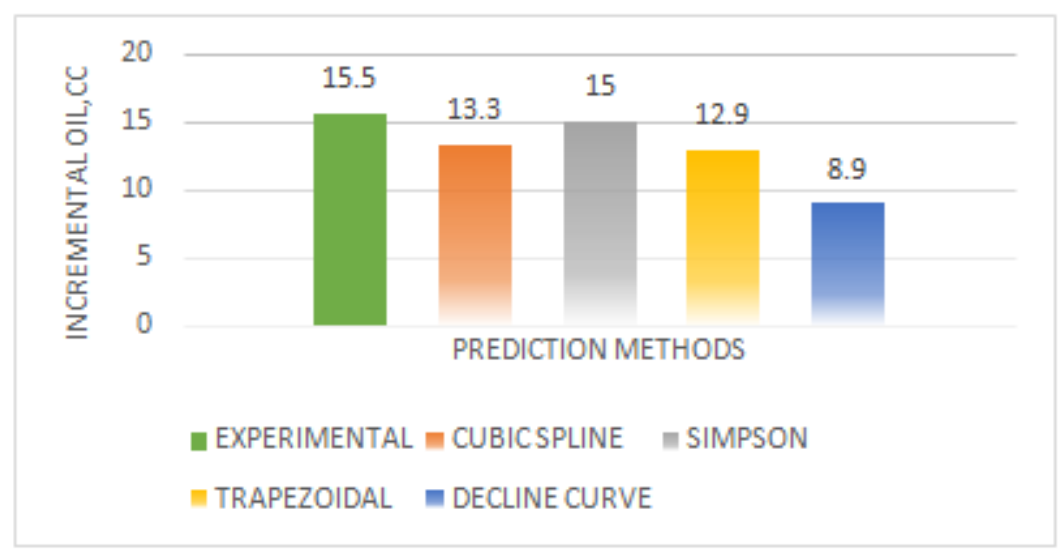

Figure8. Incremental oil prediction for the alkaline surfactant polymer flooding case study

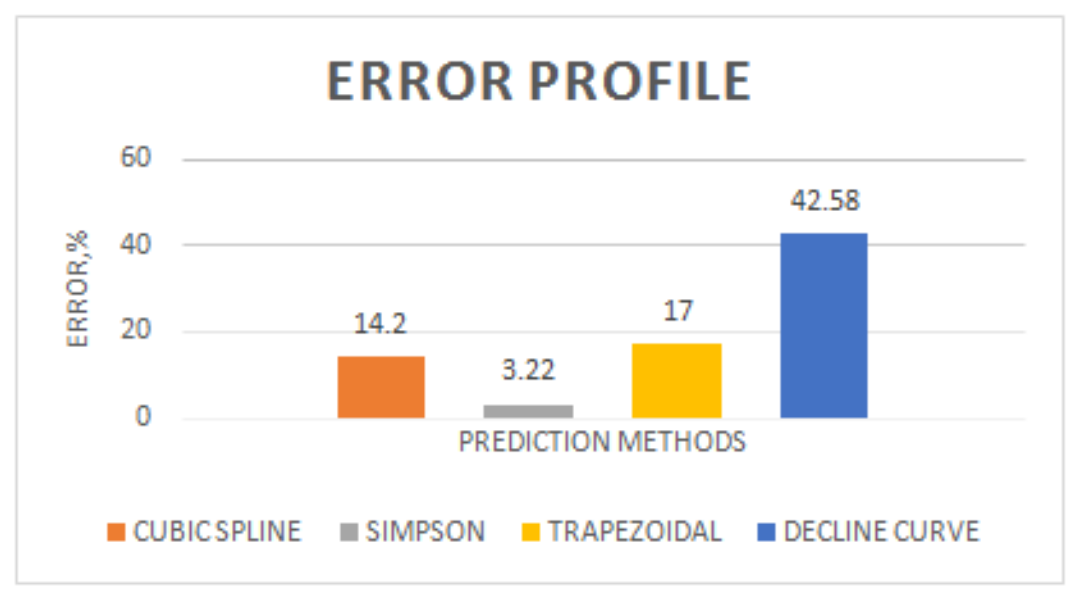

Figure8b. Error profile for alkaline surfactant polymer flooding case study.

The figures 6( $a$ and $b), 7(a$ and $b)$ and $8(a$ and $b)$ clearly shows the superiority of the Natural cubic spline and Simpson quadrature in predicting incremental oil from successful EOR processescompared to the existing methodologies. This is shown clearly in the "error profile" plotswhere the Natural cubic spline and Simpson rule out-performed the Decline curve and the Trapezoidal rule.

One of the reasons for this better peroformance of the Numerical methods can be attributed to the adoption of higher degree polynomials in modelling data points. In Natural cubic spline,a cubic polynomial is used to numerically model between two knot points while the Simpson's has been shown to be exactly accurate for some cubic polynomials. This better model data points compare with Trapezoidal rule and totally different from the concept Decline curve adopts

It is also worthy of notethat the introduction of Finite difference, in this research work has effectively helped in accounting for the error term in both the Simpson's rule and trapezoidal rule and has improved the result of both when compare to earlier work especially for the Trapezoidal rule.

\section{CONClusion}

From the analysis of the results, the following conclusion can be drawn;

1. The amount of incremental oil predicted by the Natural cubic spline and Simpson's rule were more accurate than that of the Trapezoidal rule and Decline curve.

2. The error due to trapezoidal can be attributed to its assumption of linear relationship between data in approximation given data points, which is hardly obtainable in real field data

3. The introduction of Finite difference effectively helps account for the error terms in both trapzoidal rule and Simpson's rule respectively and consequently emhanced their accuracies.

4. The Cubic spline and simpson's rule both represent a viable Numerical tool and gives a reliable prediction of incremental oil regardless of the number od data points considered 
5. The difference betwwen the integral under the incremental oil recovery curve and the integral under the corresponding curve of the projected decline represents the incremental oil.

6. Using higher degree polynomial,as adopted by the Natural cubic spline and Simpson rule to approximates data points effectively and therefore when integrated gives a better incremental oil.

\section{NOMECLETURE}

$\begin{array}{ll}\text { EOR } & \text { Enhanced oil recovery } \\ x & \text { Independent variable } \\ x_{i} & \text { Node } \\ f_{i, i+1} \text { and } f_{i, i-1} & \text { Interpolant or cubic } \\ f_{i, i+1}^{\prime} & \text { First derivatives } \\ f_{i, i+1}^{\prime \prime} & \text { Second derivatives } \\ \Delta f_{i} & \text { First forward difference } \\ N_{p} & \text { Cumulative production }(c c) \\ q & \text { Recovery rate }(c c / m i n) \\ y_{i} \text { Knot } & \\ k_{i} & \text { Coefficient of the second derivatives } \\ i, I, J & \text { Position vectors } \\ M & \text { Position vector } \\ h & \text { Height (mins) } \\ \frac{d^{2} y}{d x^{2}} \text { Second order differentials } \\ \frac{d^{4} y}{d x^{4}} \text { Fourth order differentials }\end{array}$

\section{REFERENCES}

[1] Prats M, (1982). Thermal recovery. New York: SPE of AIME; 1982.Google Scholar .

[2] J. M. Campbell, (1960). Oil Property Evaluation, Prentice Hall, Inc, Englewood Cliffs, 1960

[3] Steven, C., Chapra, Raymond, P. C., (2010), "Numerical Methods for Engineers," Sixth Edition. Tufts University and University Of Michigan.

[4] Onuoha S.O. SPE, Olafuyi O.A SPE.(2013). Alkali/Surfactant/Polymer flooding using Gum Arabic; A comparative analysis. This paper was presented at the Nigeria Annual International Conference and Exhibition held in Lagos, Nigeria, 30 July-1 August 2013.Orivri, D.U, Taiwo, O.A. and Olafuyi, O.A. (2014). "Characterizing wettability Effect on Recovery from Surfactant Flooding in a light Oil Porous Media". The Journal of Nig. Institution of Prod. Engineers, Vol. 17, Page 143-152, June, 2014

[5] Atsenuwa J.B. Taiwo O.A., Mohammed I.U., Dala, J.A and Olafuyi, O.A, (2014). "Effect of Viscosity of Heavy Oil (Class-A) on Oil Recovery in SP Flooding Using Lauryl Sulphate and Gum Arabic”. SPE 172401, Presented at the Nigeria Annual International Conference and Exhibition (SPE-NAICE), Lagos, August 5-7, 2014.

[6] Yebu W. Y. F, Taiwo O. A and Olafuyi O. A, (2015). University of Benin, "Oil Recovery Performance of Surfactant/Polymer solutions in Flooding (A Comparative study)". This paper was presented at the $1^{\text {st }}$ International Conference on Oilfield Chemistry \& Flow Assurance (Oilflow 2015) at CPRT UNIPORT, Port Harcourt, Nigeria 22 - 24 June, 2015

[7] Uwari F, SPE, Taiwo O.A, SPE, Olafuyi O.A ,SPE, (2015). University of Benin. Nigeria. "SurfactantPolymer Flooding Schemes (A Comparative Analysis)". Presented at the Nigeria Annual International Conference and Exhibition (SPE-NAICE) in Lagos, Nigeria, 4 - 6 August 2015

[8] Kharma R, SPE, Taiwo O. A, SPE, Olafuyi O. A, SPE, (2015) University of Benin, "Flooding For Light Oil Recovery In Strongly Water Wet Cores Using Sodium Hydroxide, Lauryl Sulphate, And Gum Arabic". Presented at the Nigeria Annual International Conference and Exhibition (SPE-NAICE) in Lagos, Nigeria, $4-6$ August 2015 
[9] Taiwo O. A and Olafuyi O. A. (2015), "Surfactant and Surfactant-Polymer flooding for light oil: a gum Arabic approach", Petroleum and coal Journal, 57(3) 205-215, 2015. (http://www.vurup.sk/volume-572015-issue-3)

[10] O. Taiwo, K. Bello, I. Mohammed, O. Olafuyi, (2016). "Characterization of Surfactant Flooding for Light Oil Using Gum Arabic", International Journal of Engineering Research in Africa, Vol. 21, pp. 136-147, 2016

[11] Mamudu Abbas, Taiwo Oluwaseun and Olafuyi Olalekan, (2016). "Cubic Spline And Graphical Techniques For Determining Unsteady State Relative Permeabilities In Field Cores", a paper presentedat the SPE Nigeria Annual International Conference and Exhibition (SPE-NAICE), Lagos,Nigeria, August. (2016).

[12] Sean O. Onuoha, Olalekan Olafuyi and Oluwaseun Taiwo, (2017). "Grain Size Effect on Permeabilty and Pressure Drop inAlkali-Surfactant-Polymer Flooding" a paper presented at the SPE Nigeria Annual International Conference and Exhibition (SPE-NAICE), Lagos, Nigeria, August. 2017.

[13] Ojukwu Izuchukwu, Taiwo Oluwaseun Ayodele,Gimba S. B. Abdullahi,Dala Joshua, and Olafuyi Olalekan, (2018). "Visualization of Heavy Oil Recovery Processes Using Hele-Shaw Cell", a paper presented at the SPE Nigeria Annual International Conference and Exhibition (SPE-NAICE), Lagos, Nigeria, August. 2018.

[14] Madugu Abdulraheem, Tukur Hamisu, Gimba Abdullahi, Taiwo Oluwaseun, Bello Kelani, Ismail Mohammed and OlafuyiOlalekan, (2018). "Comparative Analysis on Rate Dependent Polymer Flooding Using Bio and Synthetic Polymers"a paper presented at the SPE Nigeria Annual International Conference and Exhibition (SPE-NAICE), Lagos, Nigeria, August. 2018.

[15] Taiwo Oluwaseun, Bello Kelani and Olafuyi Olalekan, (2019). Polymer Injection Performance in Multi Porous Medium International Journal of Petroleum and Petrochemical Engineering 2019, 5(3) : 10-16

[16] Sky McKinley and Megan Levine, (2012). Cubic Spline Interpolation. Heriot-Watt Course Title ENG 101 Notes, Uploaded By ibrahimsadek87. 2012.

[17] Mamudu Abbas, Taiwo Oluwaseun and Olafuyi Olalekan, (2016). "Cubic Spline and Graphical Techniques For Determining Unsteady State Relative Permeabilities in Field Cores", a paper presentedat the SPE Nigeria Annual International Conference and Exhibition (SPE-NAICE), Lagos,Nigeria, August. 2016.

[18] Jerome, F., Trevor, H., Robert, T., (2001). "The Element of Statistical Learning, Basis Expansion and Regularization," pp. 115-164.

Citation: Oloyede Olaoye, et.al, (2019). “Application of Numerical Methods for the Analysis of Incremental Oil”, International Journal of Petroleum and Petrochemical Engineering (IJPPE), 5(4), pp.14-22, DOI: http://dx.doi.org/10.20431/2454-7980.0504003

Copyright: (C) 2019 Authors. This is an open-access article distributed under the terms of the Creative Commons Attribution License, which permits unrestricted use, distribution, and reproduction in any medium, provided the original author and source are credited 